\title{
A Short Walk along the Gravimeters Path
}

\author{
Iginio Marson \\ Department of Civil Engineering and Architecture, University of Trieste, Via Valerio 6, 34127 Trieste, Italy \\ Correspondence should be addressed to Iginio Marson, marson@units.it
}

Received 22 March 2012; Accepted 1 June 2012

Academic Editor: Umberto Riccardi

Copyright ( $) 2012$ Iginio Marson. This is an open access article distributed under the Creative Commons Attribution License, which permits unrestricted use, distribution, and reproduction in any medium, provided the original work is properly cited.

The history of gravity measurements begun in 1604 with Galileo Galilei experiments on the acceleration due to the gravity force of the earth, $g$, along inclined planes. In his memory, the most used unit to measure $g$ is the gal $\left(10^{-2} \mathrm{~m} / \mathrm{s}^{2}\right)$. The paper takes the interested reader through a walk along some of the most important achievements in gravity measurements and gives some perspectives for future developments in terrestrial gravity.

\section{Introduction}

The gravity force of the earth is a physical quantity of interest for geodesy, geophysics, and metrology. It is the main force which is responsible of the structure and shape of the earth. The combined effect of gravitational attraction and centrifugal force acts to distribute less dense material in the outermost layers of the earth and denser one in the innermost ones.

The study of the shape of the earth and of its deformation is the basic topic of geodesy. To describe the shape of the earth, geodesists have chosen the equipotential surface which coincides with the surface of the mean sea level in a given epoch, the geoid. The surface of the mean sea level in a given epoch is determined by the gravity field of the earth. From the gravity field it is possible to determine the morphology of the geoid over the entire planet. Space geodesy and in particular satellite altimetry provides a direct measurement of the sea level surface over the oceans and hence the determination of the geoid. The determination of the geoid over the land is, however, possible only by means of the gravity field. The geoid is the reference surface for elevation measurements and it is therefore important for both cartography and navigation purposes. Oceanic currents are also partly controlled by the morphology of the geoid and hence a knowledge of the geoid is of importance for oceanography, hydrology, and for environmental studies related with possible changes of the mean sea level.
Geophysics is more concerned with studying anomalies in the gravity field which are due to anomalous distribution of masses within the earth. Geodynamical processes like plate tectonics, mountain building, convective motions in the earth mantle, and volcanism have all strongly changed the mass distribution within the earth from that which would have been produced simply by the combined effects of gravitational attraction and centrifugal force. The thickness and composition of the earth lithosphere are highly variable over the whole planet. This diversification generates important anomalies in the gravity field. Geophysics is particularly interested in the study of the gravity anomalies because they permit the determination of the geological structures (faults, crust-mantle boundary, density anomalies in the mantle, etc.) which are responsible of the anomalies. Gravity is therefore a basic tool for studies of structural geology. Some geological structures within the earth crust (such as faults, synclines, antisynclines, salt domes) are frequently associated with potential reservoirs for oil and gas. As a result, the study of the Earth's gravity field also plays an important role in the prospection for oil as well as for geothermic.

In Metrology, a knowledge of the gravity field is used as a transfer standard in the determination of metrological standards involving force and pressure measurements. Therefore gravity is required in connection with applications of the electrical standards to a nonartifact standard of mass (i.e., a new Kilogram standard) as well as the calibration of pressure gauges and weighting devices. Metrological use of gravity as a constant, since it varies with both position and time, 
requires that it is measured wherever it is needed, and, if the accuracy requirements are higher than $10^{-8}$, it will be necessary to measure it also at the time it is needed. The definition of reference gravity networks to provide reference sites for metrological, geodetic, and geophysical purposes at different scales (from national to continental to global) is a topic for geodetic metrology. The main activities of this branch of metrology are the determination of gravity datum and the verification of the gravity scale. The accuracy requirements differ considerably and are strongly related to the purpose of the research. Metrology, related to standards of force and pressure, needs to know $g$ with an accuracy of the order of $10^{-6}$. The same order of magnitude is also required by geophysical studies aimed at the definition of deep and broad geological structures (for instance, the crustmantle boundary). For shallower structures (faults within the earth crust) the accuracy requirements are about one order of magnitude higher, $10^{-7}$. This order of magnitude is also required for geoid determination. Modern gravimetry allows for the determination of gravity anomalies with accuracy of the order of $10^{-8}$ which are of importance for civil engineering and archeology. This high accuracy allows also for the determination of time-dependant gravity anomalies related with motion of fluids within the earth crust, such as geothermal fluids, and changes in the water table or density variations due to geodynamical processes. Force metrology, as it will applied to the replacement of the kilogram, will require an accuracy between $10^{-8}$ and $10^{-9}$. Stationary gravimeters of high accuracy, $10^{-10}$, are used to measure temporal variations of the gravity field at a given site with the principal target of this research being the determination of the Earth's response to tidal forces. Different applications require not only different accuracy but also different special and temporal sampling. Typically, the spacial density of measuring sites ranges from one site every $10 \mathrm{~km}$ for deep geological structures, to one site every $0.5 \mathrm{~km}$ for prospection for oil and gas, to one site every $20 \mathrm{~m}$ for civil engineering.

Anomalies in the earth gravity field can be also determined by studying the perturbations in the orbits of artificial satellites. The long-wavelength part of the earth's gravity field from space as determined from various tracking measurements of earth orbiting satellites was of considerably varying quality and of incomplete geographical coverage. Consequently the accuracy and resolution of the earth gravity field models were poor, with most of the satellite contributions limited to wavelengths of $700 \mathrm{~km}$ or longer. Only broad geophysical features of the earth's structure could be detected. An impressive progress in satellite gravimetry is due to the GRACE twin satellites mission, Gravity Recovering and Climate Experiment, launched by NASA in March 2002, which has made detailed measurements of earth's gravity field (http://www.csr.utexas.edu/grace/). More recently, the ESA mission GOCE, Gravity Field and Steady-State Ocean Circulation Explorer that was launched on March 17, 2009, aims at the determination of gravity-field anomalies with an accuracy of the order of $10^{-6}$ and of the geoid with an accuracy of about $2 \mathrm{~cm}$ and a spatial resolution better than 100 km (http://www.esa.int/SPECIALS/GOCE/).
Space geodesy techniques, principally GPS has made it possible to determine the position of a moving object with high (submeters) accuracy. This capability has made a main contribution to the solution of the key problem in the measurement of gravity from a moving platform, that is, the separation of gravitational and inertial accelerations. Ship-borne gravimetry has been carried out for several decades. In the case of sea-surface gravity measurements, the separation between gravitational and inertial accelerations is facilitated by the different wavelengths of the two signals and by the periodic character of the inertial accelerations which therefore can be removed through the use of filters. In the case of airborne gravimetry, however, the speed of the aircraft, being considerably greater than the speed of a ship, reduces the gap between the wavelengths of the two signals so that the use of filters is no longer effective. Here differential GPS has helped us to tackle this problem by providing direct measurements of the position of the aircraft as a function of time, from which, by double differentiation, the inertial acceleration can be computed. Recent experiments have demonstrated that airborne gravimetry can be used to measure the gravity field with an accuracy of about $10^{-6}$ making it possible to also use this technique for applications such as oil prospecting. Table 1 summarizes some application fields together with the related accuracy target.

\section{A Historical Walk along the Gravimeters Path}

In principle any physical process which involves gravity can be used to measure $g$. The early theory of gravity based on Aristotle (384-322 BC) principle that the velocity of a free falling body is proportional to its weight was changed by Galileo in 1604 who discovered, by means of experiment with inclined planes, that in the free fall the acceleration is constant and independent from the mass.

The measurement of $g$, an absolute value of an acceleration, implies the measurement of length and time: period and equivalent length of a pendulum or time required by a free falling object to travel a given distance, hence the need of metrological standard for length and time for high precision absolute measurement of $g$. For the measurement of difference of gravity, however, it is possible to measure only time, for example, the change of the period of a pendulum of known length or the extension of a mechanical or electromagnetic spring supporting a fixed mass. Table 2, partially compiled after Torge [1], reports the progress made in measuring the gravity acceleration.

2.1. Yesterday. The pendulum method for measuring $g$, introduced by Huygens in 1656 was first used because of its mechanical simplicity and its nearly amplitude-independent (isochronous) period of oscillation. For an ideal, small amplitude of swing pendulum, the period of oscillation is only a function of the length of the pendulum and $g$. In an ideal (mathematical) pendulum, its length is simply the distance between the point of suspension and the point at which the entire mass is concentrated. In a real (physical) pendulum, which consists of an extended mass swinging 
TABLE 1: Field of application, accuracy target, type of instruments and their availability.

\begin{tabular}{lcl}
\hline Application & Accuracy target & Instruments/availability \\
\hline Isostasy & $10^{-5}$ & Relative gravimeters (pendulum)/1817 \\
Prospection for oil and gas & $10^{-6}-10^{-7}$ & Relative gravimeters/1950 \\
Structural geology & $10^{-6}$ & Relative gravimeters/1950 \\
Microgravity prospection & $10^{-8}$ & Relative gravimeters/1980 \\
Metrology (forces) & $10^{-6}-10^{-7}$ & Absolute gravimeters/1980 \\
Metrology (kilogram) & $10^{-8}-10^{-9}$ & Absolute gravimeters/1990 \\
Geodetic metrology & $10^{-8}$ & Absolute gravimeters/1980 \\
Geodesy & $10^{-7}-10^{-8}$ & Rel + Abs gravimeters/1980 \\
Earth tide and earth dynamic & $10^{-10}-10^{-11}$ & Superconducting gravimeters/1970 \\
\hline
\end{tabular}

TABLe 2: Progress in measuring $g$ (partially compiled after Torge 1989, page 14, figure 1.1 [1]

\begin{tabular}{lccc}
\hline Type of instrument & Time span & Uncertainty range & Rate \\
\hline Reversible pendulum & 1817 to 1945 & $4 \times 10^{-5}$ to $2 \times 10^{-6}$ & One order of magnitude/130 years \\
Spring type gravimeters & 1935 to 2010 & $4 \times 10^{-7}$ to $3 \times 10^{-9}$ & Two orders of magnitude/75 years \\
Superconducting gravimeters & 1965 to 2010 & $5 \times 10^{-10}$ to $5 \times 10^{-12}$ & Two orders of magnitude/45 years \\
Absolute gravimeters & 1970 to 2010 & $8 \times 10^{-7}$ to $2 \times 10^{-9}$ & Two orders of magnitude/40 years \\
\hline
\end{tabular}

about a certain point of suspension, its length depends on the mass distribution of the pendulum relative to the point of suspension. To achieve an accuracy of the order of $10^{-6}$, the length of the pendulum must be measured with an accuracy of the order of $10^{-6}$ and the period of oscillation with an accuracy two times better than this. Because of the isochronous character of the oscillation, the accuracy in the measurement of the period can be increased by measuring the time for a number of oscillations. However, in spite of its conceptual simplicity, the main problem with pendulum measurements remains with the determination of the "length". Nevertheless, the measurement of $g$ begun with, and exclusively depended on, pendulum measurements until the end of nineteenth century. Figure 1 shows an example of a pendulum.

Pendulum measurement played a role in the discussion between Cassini and Newton concerning the flattening of the earth. In 1672 the French astronomer Jean Richer had measured the period of a pendulum at Paris and at Cayenne, which is almost on the equator. The gravitational force was found to be less in Cayenne than in Paris, a discrepancy that G. D. Cassini, the first of a dynasty that was to dominate French observational astronomy throughout the eighteenth century, took to be evidence that there was less mass of the earth under the equator than under more northerly latitudes; that is, that the earth is not quite a sphere but is a prolate ellipsoid. (Rowlinson 2007 [2]).

Until the end of the eighteen century, all the pendulum measurements were absolute, that is, both period of oscillation and length of the pendulum were measured at a given site and g computed from these two quantities. The measurements were rather lengthy and complicated and were made mainly in laboratory conditions. Of fundamental importance for the history of gravimetry remains the absolute measurement of $g$ made by Kuhnen and Furtwangler [3] between 1898 and 1904 in Potsdam which was used as the base for the Potsdam Gravity System introduced in 1908 and extended worldwide. In spite of the duration of the measurements, the Potsdam datum had a significant systematic error which was discovered only several years later. In 1817, Kater invented the reversible pendulum [4]. Consider a pendulum which can be hung from either of two points. If the period of swing is the same about each of the two points of support, then the distance separating the two points of suspension is equal to the length of a simple pendulum having the same period. Thus the metrology is reduced to measuring the period of oscillation and the distance between the two supports. Pendulum measurements are affected by several sources of errors, the main ones being: the vibration of the pendulum support, the change in the pendulum length and the influences of nongravitational forces (friction, magnetic and electrostatics). A comprehensive overview of the development of instruments, measuring techniques and application is given in Nabighian et al. [5].

During the first half of the twentieth century several improvements were made on pendulums: apparatus with two or four pendulums, generally made by quartz to minimize temperature dependent effects, were manufactured to reduce sources of errors. Pendulums both as absolute and relative measurements have been used in various configurations until about 1940, both for laboratory and field observations in land, on ships and underwater. Kater has also the merit to have recognized that for the geophysical needs of gravimetry, detection of anomalies in the gravity field of the Earth, it is sufficient to measure the changes in gravity with the position, hence diminishing constraints in the physical parameters to be measured and increasing the accuracy. For instance, provided that the "length" of the pendulum remains constant during the measurements, the gravity differences between two observations points are related to the difference in the periods of oscillations. Relative gravimetry was born after this achievement. 


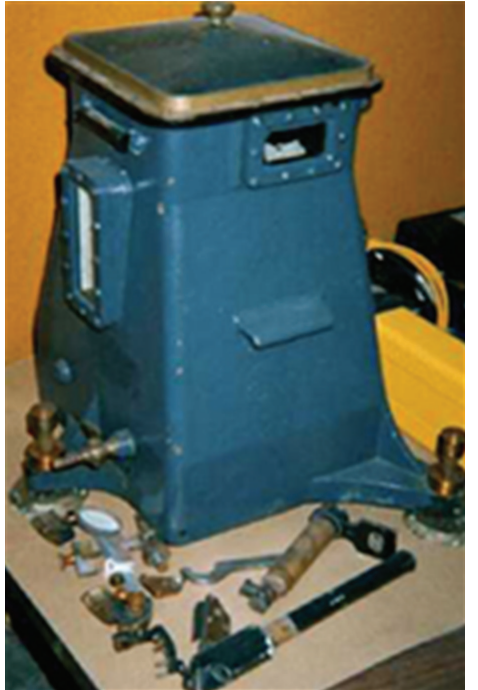

Figure 1: The Mendenhall pendulum.

A significant breakthrough in gravity measurements resulted from the introduction of spring-type relative gravimeters. These instruments are basically weighing devices in which the force on a mass due to gravity is balanced by the restoring force of a spring. Historically the spring-type gravimeters can be divided in two types: linear, in which the equilibrium conditions are between two forces (elastic and gravitational) and astatic, in which a condition close to equilibrium is reached by equating the momentum of the gravitational and elastic forces. The great majority of the modern gravimeters belongs to the second category. The advantage of astatic gravimeters over linear ones is that their sensitivity can be greatly increased through the use of astatization where a small change in force results in a large change in position. Gravimeter springs are normally made of metal or fused silica. Metal leaf, coiled, and helical springs have been used in different type of gravimeters. A key problem with metal springs is the strong dependency of their elasticity on temperature. Usually this type of gravimeters requires a well-defined operating temperature. A natural counterpart to metal is fused silica. Springs made of fused silica are rather strong and slightly less sensitive to temperature. On the other hand this type of gravimeters shows a large linear drift due to evaporation of silica in its container under vacuum. Sam Worden developed in in the late 1940's the Worden Gravity Meter based on this principle. Another problem which affects spring-type gravimeters is related to pressure compensation, that is obtained either by a pressure compensation box mounted on the beam opposite to the mass with respect to the rotation axes or by putting the instrument in a sealed container. As a third category of "spring" some gravimeters use gas at a particular temperature and pressure as the spring element. Gas-type gravimeters were employed in the '30ies mainly as shipborne gravimeters because the high degree of damping which resulted from the use of a gas spring which made this kind

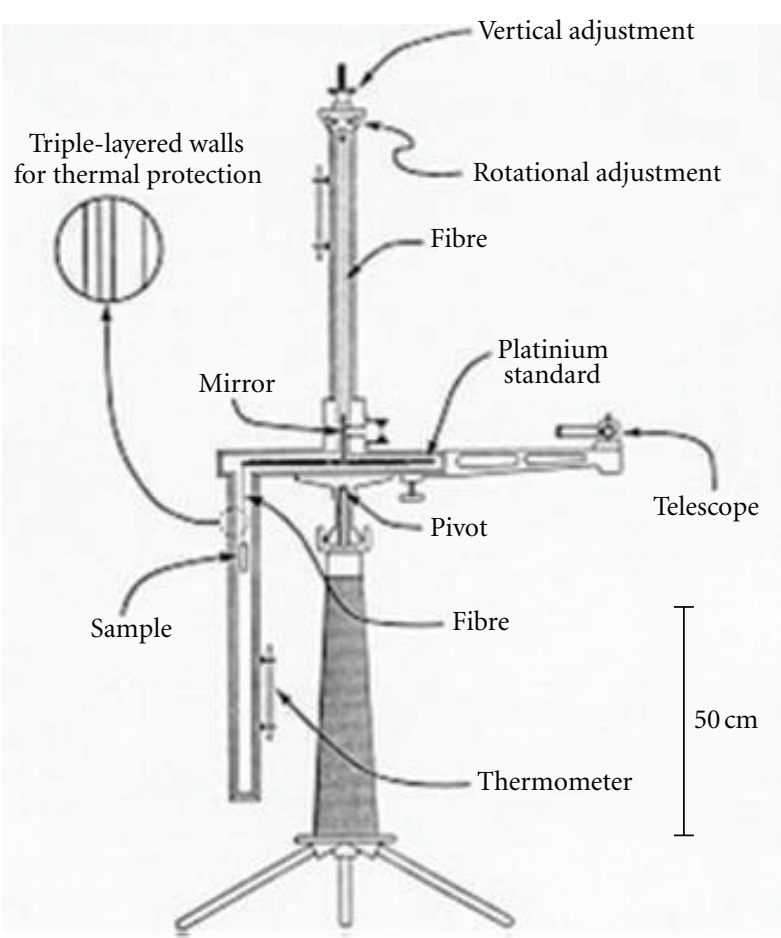

Figure 2: Eotvos Torsion balance.

of instruments less sensitive to vibrations and hence better suited for measurements on the sea surface.

A peculiar kind of gravity meter, used between the two World Wars, is the torsion balance developed by Baron Roland von Eotvos in 1896. The torsion balance consists of a torsion platinum-iridium fiber suspended vertically with a horizontal aluminum bar suspended from its lower end. The proof mass is positioned on one end of the bar, while on the other end there is an identical proof mass suspended by another fiber several centimeters below the horizontal plane of the bar. If the gravity field of the earth at the measurement point is distorted by an anomalous mass at depth, the balance bar rotates since the horizontal component of gravity at one test mass is different from that at the other test mass. The magnitude of the torque can be determined by measuring the angle through which the balance has been rotated by the torque. Figure 2 shows a schematic of a Torsion balance.

A second break-through in relative gravimetry resulted from the introduction of the zero length spring. For this type of spring, the restoring force is proportional to the entire length of the spring. Its importance results from the fact that when employed in an astatic scheme, with the point of application of the spring and the pivot of the arm holding the test mass located on the same vertical line, the equilibrium condition is independent from the deflection angle of the arm, hence making the sensitivity (in principle) infinite. Since 1934 when LaCoste [6] made their first gravity meter based on the zero length spring (which was actually born as long period seismometer), this type of gravimeter has dominated the scene of relative gravimetry for something like 
50 years. Figure 3 shows a schematic diagram of a LaCosteRomberg gravity meter.

The principal problem with spring-type gravimeters is the elastic variability of the spring. All these kinds of gravimeters suffer because of anelastic drift which depends on time as well as temperature changes and the effects of mechanical shocks and vibrations. The discovery of superconductivity in certain materials at low temperature resulted in a new kind of "spring-type" gravimeters which is, at least in principle, drift free. In the superconducting gravimeter, the test mass, a metallic sphere made from or coated with a layer of superconducting metal, is levitated by the magnetic field generated by current flowing in a superconducting coil. The instrument, a stationary one, is installed at a fixed site and measures gravity variations with time. The measured changes in current necessary to keep the test mass in the reference position are proportional to the variations in the gravity field as results of tides, variations of barometric pressure and local atmospheric, mass movements, or density variations within the earth. Superconducting gravimeters, invented by Prothero and Goodkind [7] in the mid '60ties, provide a very high sensitivity of the order of 10-10 and, owing to the superconducting properties, can be nearly drift free.

Relative gravimeters all require a calibration in order to determine what changes of spring length or of electrical current correspond to a given gravity change. Relative gravimeters have been widely employed to make measurements on land, on the sea surface, at the sea bottom and, recently, from the air. All geophysical problem as well as several geodetic problems can be solved using relative techniques. The basic problem of geodetic metrology (definition of gravity datum and of gravity scale), however, cannot be solved using relative gravimetry. It was just after the second world war, when techniques for high-accuracy measurements of time and space intervals became available, that measurements of $g$ through studying the motion of a free falling object became possible. A pendulum's advantage with respect to studying a free falling object is that, in the case of pendulum, the accuracy of the time measurement can be simply increased by increasing the number of oscillations. This is not possible in the case of a free fall experiment; on the contrary, in this case, it is necessary to measure with high accuracy small time intervals. Fortunately our ability to do this has increased dramatically over the past 3-4 decades. As a result, the second half of the 20th century has seen the development of free fall methods for the absolute determination of $g$. During this period, a number of absolute determinations used the method of geometrical optics to define the position of the falling object, while during the past 30 years the method of laser interferometry has been used. Using laser interferometry to measure distances has been a key point in the development of today's transportable absolute gravity meters: it permits the measurement of distances of the order of $1 \mathrm{~m}$ with the accuracy of $1 \mathrm{~nm}$ during the subsecond timescale associated with a single drop. A comprehensive overview of this historical period of absolute gravimeters is given in Marson and Faller 1986 [8].
After the first instruments which used geometrical optics (Martsyniak1956 [9], Preston-Thomas et al. 1960 [10] and Thulin, 1960 [11]), Faller, 1962 had developed the first free fall interferometric instrument (Faller 1965 [12]). Meanwhile Sakuma at Bureau International des Poides et Mesures, Sevres, France [13] was working on a rise and fall interferometric instrument. When lasers with frequency stabilization became available, Hammond and Faller (1967) [14] developed the first transportable absolute gravity meter of the free fall type. A few years later, Hammond (1970) [15] modified and made improvements of this transportable meter. Both, the initial and modified instruments used multiple stations coupled with laser interferometry. Based on the principle of Sakuma's instrument the Italian Metrology Institute "G. Colonnetti" was developed in 1974 [16] in cooperation with BIPM, a portable version of the stationary apparatus (Figure 4). In this instrument, laser interferometry was applied to measure position in space. As in Sakuma's instrument, the moving object followed a rise-and-fall trajectory.

For several years, the advantages and disadvantages of these different approaches, direct free fall and rise and fall, have been debated. The free fall type of ballistic gravimeter is in principle simpler from a mechanical point of view with respect to the rise-and-fall one. This is mainly because the verticality of the trajectory in the second type strongly depends on being able to successfully throw the object upwards starting with a large release velocity, whereas in the free fall the release velocity is essentially zero. The symmetry of the trajectory on the other hand eliminates certain frequency-dependent errors in the fringe signal. Moreover, the symmetry also compensate the air drag effect due to residual pressure, diminishing the level of vacuum required for the experiment. Due to drag g seems larger at the rise and smaller at the fall. A rise and fall experiment requires a particular care in setting and monitoring the verticality of the trajectory, while the free fall one is less sensitive to this. Hence a free fall experiment can be made automatic and controlled by computer in a easier way with respect to a rise and fall one. In a measuring session, free fall instruments can perform thousands of drops while rise and fall ones almost hundred. The first are computer controlled, the second are under the control of human beings with all the plus and minus related to it.

In order to establish the accuracy of absolute gravimeters, and eventually detect sources of systematic errors, eight intercomparison campaigns have been realized at the BIPM laboratories in Sevres France. Seven instruments took part in the first campaign in 1981. All instruments were developed in specialized institutes, mainly metrological institutes or laboratories related to them, and were operated by the scientists and technicians who developed them and hence knew "every single screw". Twenty two instruments participated in the 8th campaign, the last at BIPM. The rapid increase of absolute gravimeters is due to the excellent work of Faller who developed, with his $\mathrm{PhD}$ students Niebauer [17] and Rinker [18], 6 absolute gravimeters, known as JILAg, within a scientific framework with six geodetic/geophysics institute in the USA and Europe and made available to a wider body of scientists these kind of instruments [19] (Figure 5). 


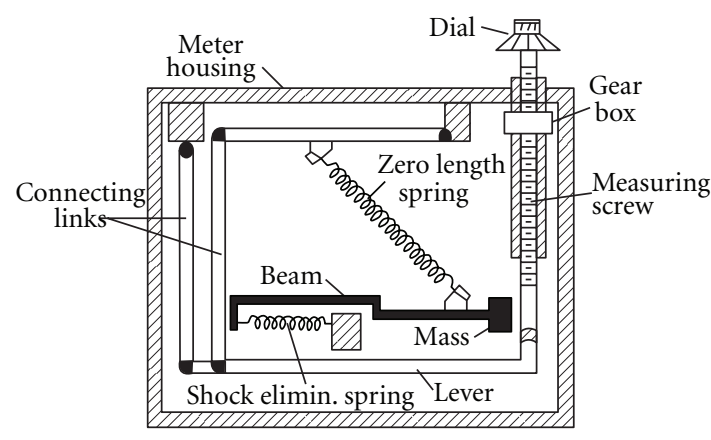

(a)

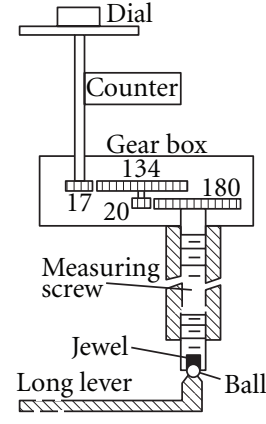

(b)

FIgURE 3: Schematic of a La Coste-Romberg gravity meter.

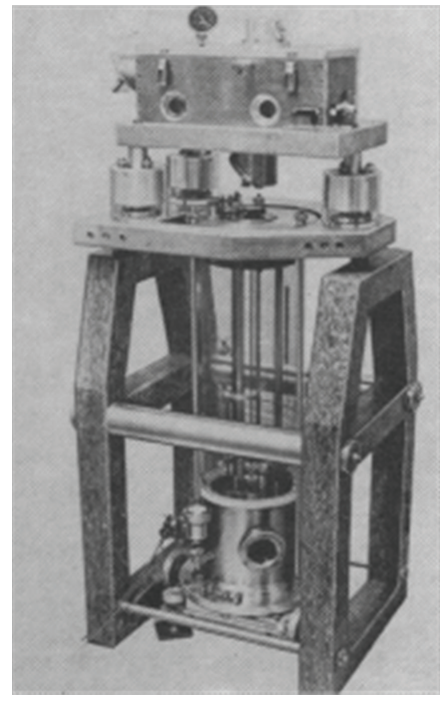

Figure 4: The IMGC absolute gravity meter.

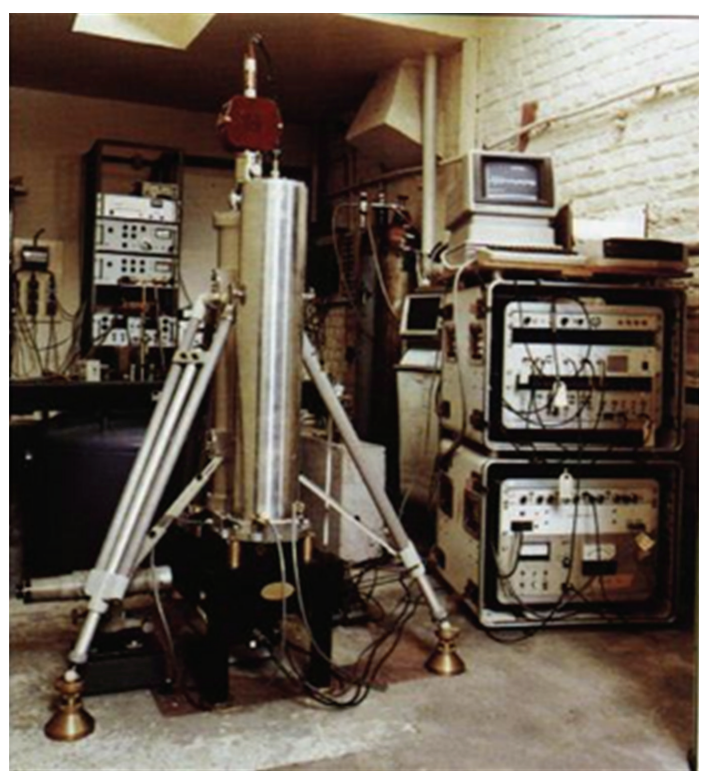

FIGURE 5: The JILAg absolute gravity meter.

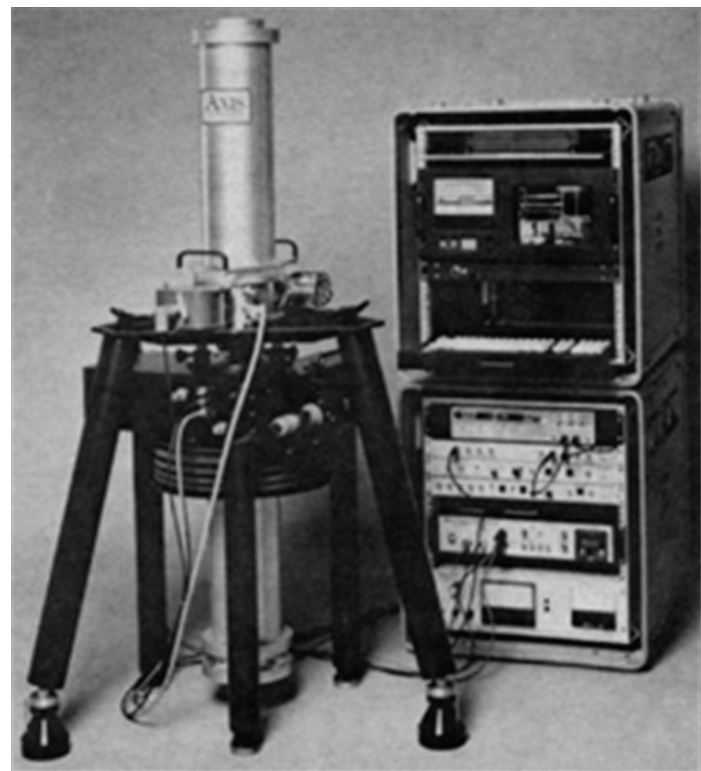

FIgURE 6: AXIS FG5 absolute gravimeter.

After Faller, T. Niebauer developed, on a commercial base (AXIS, Microg Solutions, MicrogLaCoste), a successful series of instruments, FG5, with a worldwide distribution. All these instruments are based on the free fall principle (Figure 6).

2.2. Today. The terrestrial relative gravity world is dominated for several decades, since 1939, by LaCoste\&Romberg gravity meters (Mod D and Mod G) and has witnessed in the last years the growth of Scintrex Autograv (CG3, CG5) (http://www.scintrexltd.com/gravity.html), Microg LaCoste (gPhone), http://www.microglacoste.com and ZLS (Burris), http://www.zlscorp.com. The long lasting superiority of LaCoste instrument was also due to the introduction of a capacitive transducer to monitor electronically the position of the beam in a much more impersonal way than the optical readout. It opened the way to the introduction of "feedback" electronics in order to keep always the beam of instruments at the same position (zero method). This technique was 
introduced first on the tidal LaCoste (ET meter) used for tidal registrations. The first attempt based on the use of a step motor to drive the micrometer was not very successful. Later an electrostatic restoring force has been used successfully. All instruments have nowadays electronic levels, electronic readout, and computer-controlled data acquisition. The reading sensitivity is of $1 \times 10^{-9}$, the field repeatability around $5 \times$ $10^{-9}$. The development of computer-controlled data acquisition and to certain extent also error compensation has certainly increased the instrumental reliability. As counter fact, the interaction between operator and gravity meter, which was inherent with the original instruments, has been lost.

Since the early days at the beginning of ' 80 ties, absolute gravimetry has been subjected to continuous upgrade and developments, mainly due to the continuously increased R\&D at Micro- $g$ Solutions first and, since 2005, at Micro- $g$ LaCoste. The original FG5 instrument, already an excellent instrument, has been followed by the FG5-X with a new dropping chamber and an extended free fall length of $36 \mathrm{~cm}$ within $260 \mathrm{~ms}$, by FG-L, a simplified version of FG5 and A10, a smaller version of FG5 intended for field measurements. Declared accuracy of the order of $10^{-9}$ for FG5 and FG5$\mathrm{X}$ and $10^{-8}$ for FG-L and A10. Reading the report of the Intercomparison Campaign of Absolute Gravimeters, held in Sevres, France at the BIPM laboratories in 2005, Jiang et al. 2011 [20] we clearly see that the absolute gravity world is dominated by free-fall-type instruments (FG5, A10, JILAg) with just few exceptions. One of these is the new instrument developed by the Italian Institute for Metrological Research-INRIM and indicated as IMGC-02, a memory of the original IMGC instrument. The instrument features a completely redesigned launching chamber, now automatic, a Mach-Zendher interferometer and computercontrolled data acquisition [21, 22] (Figure 7).

The repeatability, of the order of $10^{-9}$ and the total uncertainty, $8 \times 10^{-9}$, are similar to those of the free fall instruments. But what is very important to stress once again is the symmetry of the trajectory and its property of cancelling most, if not all, errors dependent from the frequency of the fringe signal.

Recently, J. E. Faller together with A. L. Vitoushkin 2005 [23], have developed a new absolute gravity meter which uses a cam to create the repeated necessary motions of hold, release, catch, and lift. By using a second cam that drives an auxiliary mass, it is possible to have the center of mass of the free fall system unchanged throughout the measurement cycle avoiding therefore any soil recoil effect. The instruments are very small, weighs less than $45 \mathrm{~kg}$ and have an accuracy comparable to that of the FG5.

The intercomparison campaigns are of high importance for the metrology of absolute gravimetry: they represent the best tool to identify and possibly correct for systematic errors and state the effective level of accuracy. Since 1981 the Bureau International des Poides et Mesures, Sevres, France has hosted eight campaigns (1981, 1985, 1989, 1994, 1997, $2001,2005,2009$ ) while two campaigns (2003 and 2011) were held in Walferdange, Luxemburg at the European Center for Geodynamics and Seismology (ECGS). The increase

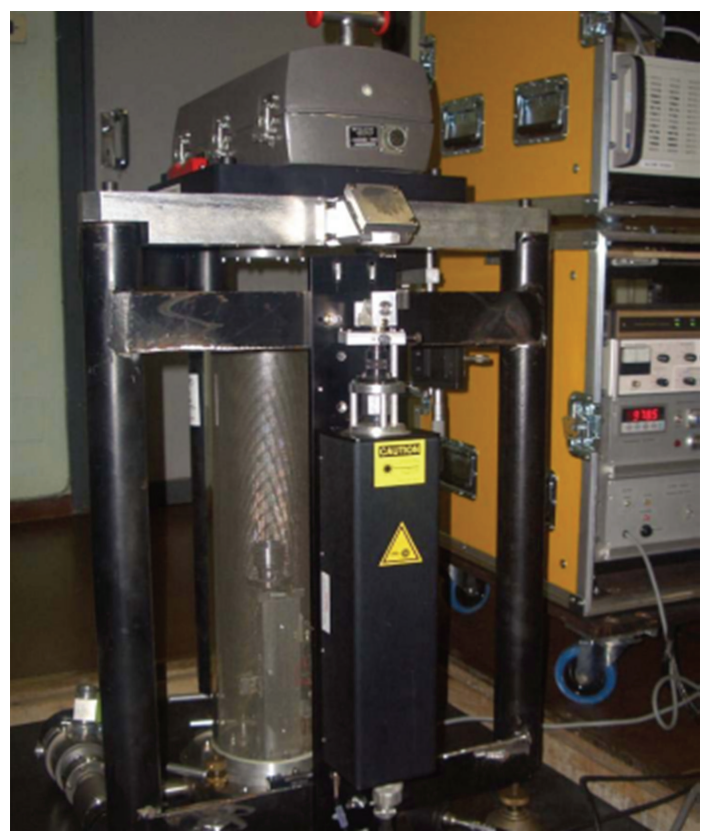

FIGURE 7: IMGC-02 absolute gravity meter.

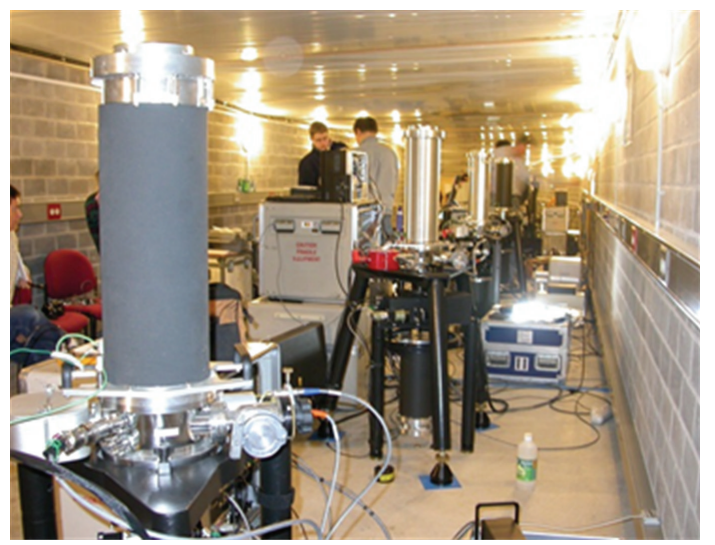

FIGURE 8: Impressive image of absolute gravimeters in "a raw" at Walferdange.

in the numbers of instruments which participate in the campaigns is impressive [20] as well as the results of the last intercomparisons which indicate an agreement between the various instruments of about $2 \times 10^{-9}$ (Figure 8 ).

As far as Superconducting gravity meters is concerned, GWR Instruments, Inc. is the exclusive manufacturer of this kind of instruments. The levitation of a spherical test mass in an ultrastable magnetic field generated by persistent currents in two niobium coils that are superconducting below a temperature of $9.3 \mathrm{~K}$ replaces the mechanical spring. As a result, the ultrastable magnetic field, weak gradient, and operation at cryogenic temperatures eliminate the sources of noise and drift commonly found in mechanical spring gravity meters so that sensitivity of the instrument reaches the level of $10^{-12}$. GWR produces two types of SG: the GWR Observatory 
Superconducting Gravimeter and iGrav SG Meter-Simplified Superconducting Gravimeter for Portable Operation.

After 30 years of continuous development of gravity meters we now have at our disposal a powerful pool of high precision and high accuracy instruments to study our dynamic Earth. It is almost impossible to overview the high amount of scientific papers which report field of applications and results of gravity experiments. List of relevant papers on the use of superconducting gravimeters can be found at http://www.gwrinstruments.com/published-papers .html and on the use of absolute gravity meters at http://www .microglacoste.com/grav_bib.php.

\subsection{The Future of Terrestrial Gravity}

2.3.1. New Instruments. Research and development aimed at reducing the size and cost of portable absolute and superconducting gravity meters is certainly the most obvious target. The most challenging task, however, concerns the development of absolute gravimeters based on a new vision of the interferometric principle: atomic interferometry, Peters et al. 1999 [24], Peters et al. 2001 [25], Schmidt et al. 2011 [26]. To measure $g$ a cold cloud of $\mathrm{Rb}$ atoms is prepared in an ultra high-vacuum chamber to be used as a test mass. During their free fall, these atoms undergo three stimulated Raman transitions, Weiss et al. [27], Moler et al. [28] that separate, redirect, and recombine the atomic wave function, resulting in an atomic interferometer, respectively. The total phase shift between the two paths of this atomic interferometer depends on $g$ and scales with the square of the time interval between two consecutive Raman pulses. This gravity phase shift is canceled by chirping the frequency difference of the two Raman beams to compensate the time-dependent Doppler shift. The value of $g$ is therefore derived from a frequency chirp. In the paper by Louchet-Chauvet et al. [29], the authors report an intercomparison experiment between three instruments based on three different methods: free fall, symmetrical rise and fall, cold atomic interferometry. Apart from the results of the intercomparison, which showed some discrepancies and indicated that there is still work to be done, the important fact is that it shows that absolute $g$ instruments based on atomic interferometry are in our near future and can achieve accuracies comparable to those of absolute gravimeters based on optical interferometry. The fundamental step forward in this new kind of instruments is that there is no need of dropping or launching mechanisms and there are no solid bodies in free fall. On the other hand, very specialized scientists to set up and perform the experiment are required.

A challenging topic for new instruments regards the development of vertical gradiometers based on the superconducting principle, van Kann et al. [30], ballistic methods, Brown et al. [31], or atom interferometry, McGuirk et al. [32]

2.3.2. Metrology. The kilogram is the last remaining base unit of the International System of Units which is still defined by a material artifact: it is equal to the mass of the international prototype of the kilogram which since 1889 has been kept at the BIPM. The main disadvantage of this definition is that it relies on the long-term stability of the mass of a material artifact. To ensure the long-term stability of the mass unit, it is advisable to link the unit of mass to a fundamental constant. Therefore, the General Conference for Weights and Measures recommended at its 21st meeting in 1999 that "national laboratories continue their efforts to refine experiments that link the unit of mass to fundamental or atomic constants with a view to a future redefinition of the kilogram". The redefinition of the kilogram would also benefit three other base units: the ampere, the mole, and the candela, the definitions of which depend on the kilogram. The watt balance experiment links the Planck constant $\mathrm{h}$ to the mass of the international prototype of the Kilogram (IPK), that is, it measures the ratio $h / m_{\text {IPK }}$. The physical principle is balancing an electrical power with a mechanical power, the weight of a reference mass. Hence the need of high-precision measurement of $g$ which can be achieved only by means of the modern absolute gravimeters based on different principles: free fall rise-andfall and atom interferometry Louchet-Chauvet et al. [29]

2.3.3. Moving Platform. As already noted, the achievements of satellite positioning systems (GPS, GNNS, GALILEO) made it possible to measure the gravity field by means of airborne gravimeters. Baumann et al. have shown [33] the potential applications of ballistic absolute gravimeters also in these kind of experiments. A step forward in this direction could be also the development of absolute vertical gradiometers due to their intrinsic property of reducing, if not eliminating, inertial accelerations.

\section{Conclusions}

The second half of the last century has witnessed a rapid increase of precision and accuracy of the gravity meters, from $10^{-6}$ at the beginning of the century to $10^{-9}$, both in absolute and relative instruments, and up to $10^{-12}$ for superconducting gravimeters in specific measurement conditions.

New type of gravimeters has been developed among which the most relevant ones are based on cold-atom interferometry which opens up a new way to perform the free fall experiment. The combination of different instruments allows for the "best as possible" measurement of $g$ when high accuracy is needed. The colocation of superconducting relative gravimeters of high sensitivity with absolute gravity meters has opened up new perspectives in the study of geodynamical processes in the earth (evolution of the lithosphere, isostatic responses to mass changes, density changes due to thermal cooling and heating, lithospheric slab pull).

\section{Disclosure}

In the paper the author has mentioned some commercial identities manufacturers of gravity meters. The author declares that he/she, as author of the paper, does not have any direct financial relation with the commercial identity mentioned in the paper that might lead to a conflict of interest. 


\section{References}

[1] W. Torge, Gravimetry, Walter De Gruyter, Berlin, Germany, 1989.

[2] J. S. Rowlinson, "Flattening the earth," Notes and Records of The Royal Society, vol. 61, no. 1, pp. 67-68, 2007.

[3] F. Kuhnen and P. Furtwangler, Bestimmung der absoluter Grösse der Schwerkraft zu Potsdam mit Reversionspendeln, vol. 27 of Veröffentlichung des Königl. preussischen geodätischen Institutes, 1906.

[4] H. Kater, "An account of experiments for determining the length of the pendulum vibrating seconds in the latitude of London," Philosophical Transactions of the Royal Society of London, vol. 108, pp. 33-102, 1818.

[5] M. N. Nabighian, M. E. Ander, V. J. S. Grauch et al., "Historical development of the gravity method in exploration," Geophysics, vol. 70, no. 6, pp. 63-89, 2005.

[6] L. J. B. LaCoste, "A new type long period vertical seismograph," Physics, vol. 5, no. 7, pp. 178-180, 1934.

[7] W. A. Prothero and J. M. Goodkind, "A superconducting gravimeter," Review of Scientific Instruments, vol. 39, no. 9, pp. 1257-1262, 1968.

[8] I. Marson and J. E. Faller, "g-the acceleration of gravity: its measurement and its importance," Journal of Physics E, vol. 19, no. 1, pp. 22-32, 1986.

[9] A. Y. Martsyniak, "Opredelenje absoliutnoi velichiny uskoreniia sily trax zhesti po paderniiu zhezla v vakuume," Izmer. Tekh. Poverochn Delo, vol. 5, p. 11, 1956.

[10] H. Preston-Thomas, L. G. Turnball, E. Green, T. M. Dauphice, and E. H. Kalra, "An absolute measurement of the acceleration due to gravity at ottawa," Canadian Journal of Physics, vol. 38, pp. 824-852, 1960.

[11] A. Thulin, "Acceleration due to gravity at the national bureau of standards," NBS Monograph, vol. 107, pp. 1-20, 1960.

[12] J. E. Faller, "Results of an absolute determination of the acceleration of gravity," Journal of Geophysical Research, vol. 70, pp. 4035-4038, 1965.

[13] A. Sakuma, "État actuel de la nouvelle détermination absolue de la pesanteur au Bureau International des Poids et Mesures," Bulletin Géodésique, vol. 69, no. 1, pp. 249-260, 1963.

[14] J. A. Hammond and J. E. Faller, "12.5-Laser-interferometer system for the determination of the acceleration of gravity," IEEE Journal of Quantum Electronics, vol. 3, no. 11, pp. 597602, 1967.

[15] J. A. Hammond, "A laser-interferometer system for the absolute determination of the acceleration of gravity," JILA Report, no. 103, 1970.

[16] G. Cerutti, L. Cannizzo, A. Sakuma, and J. Hostache, "Transportable apparatus for absolute gravity measurement," VDI Berichte, no. 21, pp. 49-51, 1974.

[17] T. M. Niebauer, New absolute gravity instruments for physics and geophysics [Ph.D. thesis], University of Colorado, Boulder, Colo, USA, 1987.

[18] R. L. Rinker, Super spring - a new type of low-frequency vibration isolator [Ph.D. thesis], University of Colorado, Boulder, Colo, USA, 1983.

[19] W. Torge, R. H. Röder, M. Schnüll, H. G. Wenzel, and J. E. Faller, "First results with the transportable absolute gravity meter JILAG-3," Journal of Geodesy, vol. 61, no. 2, pp. 161-176, 1987.

[20] Z. Jiang, O. Francis, L. Vitushkin et al., "Final report on the Seventh International Comparison of Absolute Gravimeters (ICAG 2005)," Metrologia, vol. 48, no. 5, pp. 246-260, 2011.
[21] G. D'Agostino, Development and metrological characterization of a new transportable absolute gravimeter [Ph.D. thesis], 2005.

[22] G. D’Agostino, S. Desogus, A. Germak et al., "The new IMGC02 transportable absolute gravimeter: measurement apparatus and applications in geophysics and volcanology," Annals of Geophysics, vol. 51, no. 1, pp. 39-49, 2008.

[23] J. E. Faller, "The measurement of little g: a fertile ground for precision measurement science," Journal of Research of the National Institute of Standards and Technology, vol. 110, no. 6, pp. 559-581, 2005.

[24] A. Peters, K. Y. Chung, and S. Chu, "Measurement of gravitational acceleration by dropping atoms," Nature, vol. 400, no. 6747, pp. 849-852, 1999.

[25] A. Peters, K. Y. Chung, and S. Chu, "High-precision gravity measurements using atom interferometry," Metrologia, vol. 38, no. 1, pp. 25-61, 2001.

[26] M. Schmidt, A. Senger, M. Hauth, C. Freier, V. Schkolnik, and A. Peters, "A mobile high-precision absolute gravimeter based on atom interferometry," Gyroscopy and Navigation, vol. 2, no. 3, pp. 170-177, 2011.

[27] D. S. Weiss, B. C. Young, and S. Chu, "A precision measurement of $\mathrm{h} / \mathrm{M}_{C s}$ based of photon recoil using laser cooled atoms and atom interferometry," Applied Physics B Lasers and Optics, vol. 59, no. 3, pp. 217-256, 1994.

[28] K. Moler, D. S. Weiss, M. Kasevich, and S. Chu, "Theoretical analysis of velocity-selective Raman transitions," Physical Review A, vol. 45, no. 1, pp. 342-348, 1992.

[29] A. Louchet-Chauvet, S. Merlet, Q. Bodart et al., "Comparison of 3 absolute gravimeters based on different methods for the e-MASS project," IEEE Transactions on Instrumentation and Measurement, vol. 60, no. 7, pp. 2527-2532, 2011.

[30] F. J. van Kann, M. J. Buckingham, C. Edwards, and R. Matthews, "Performance of a superconducting gravity gradiometer," Physica B, vol. 194, no. 1, pp. 61-62, 1994.

[31] J. M. Brown, T. M. Niebauer, F. J. Klopping, and A. T. Herring, "A new fiber optic gradiometer for 4-D absolute differential gravity," Geophysical Research Letters, vol. 27, no. 1, pp. 33-36, 2000.

[32] J. M. McGuirk, G. T. Foster, J. B. Fixler, M. J. Snadden, and M. A. Kasevich, "Sensitive absolute-gravity gradiometry using atom interferometry," Physical Review A, vol. 65, no. 3, article 033608, 14 pages, 2002.

[33] H. Baumann, E. E. Klingelé, and I. Marson, "Absolute airborne gravimetry: a feasibility study," Geophysical Prospecting, vol. 60, no. 2, pp. 361-372, 2012. 

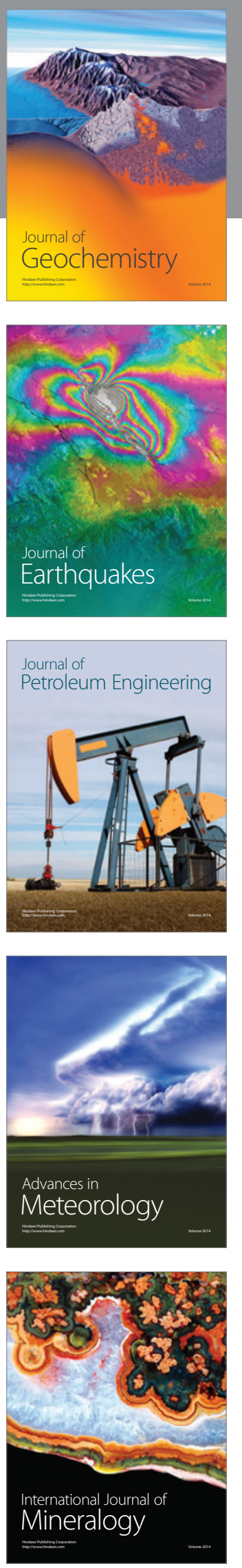
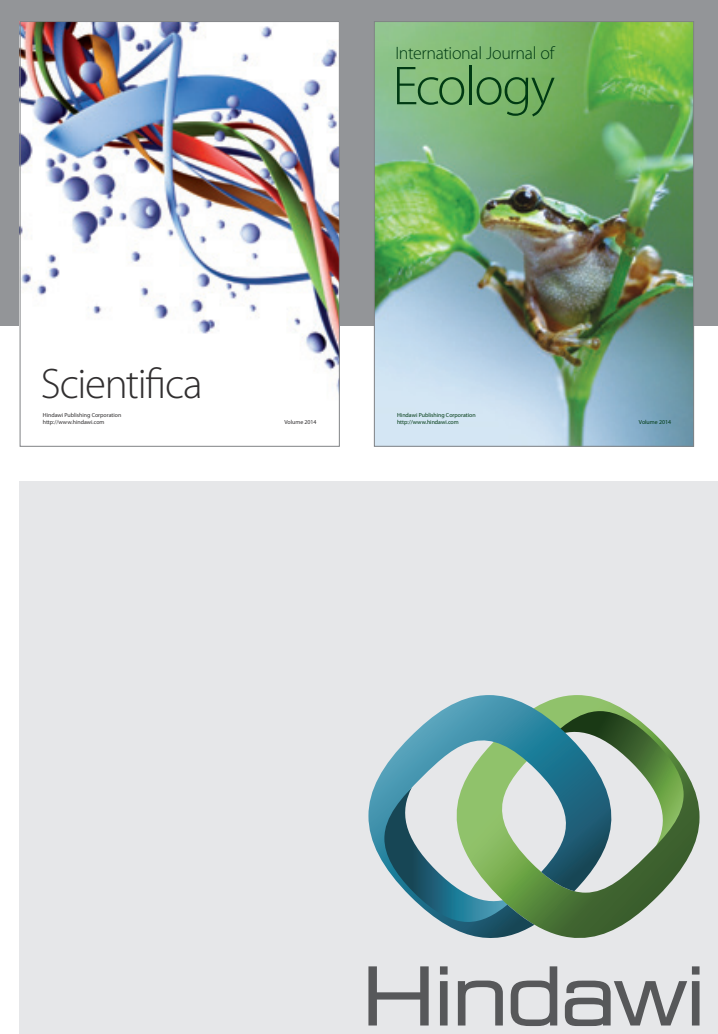

Submit your manuscripts at http://www.hindawi.com
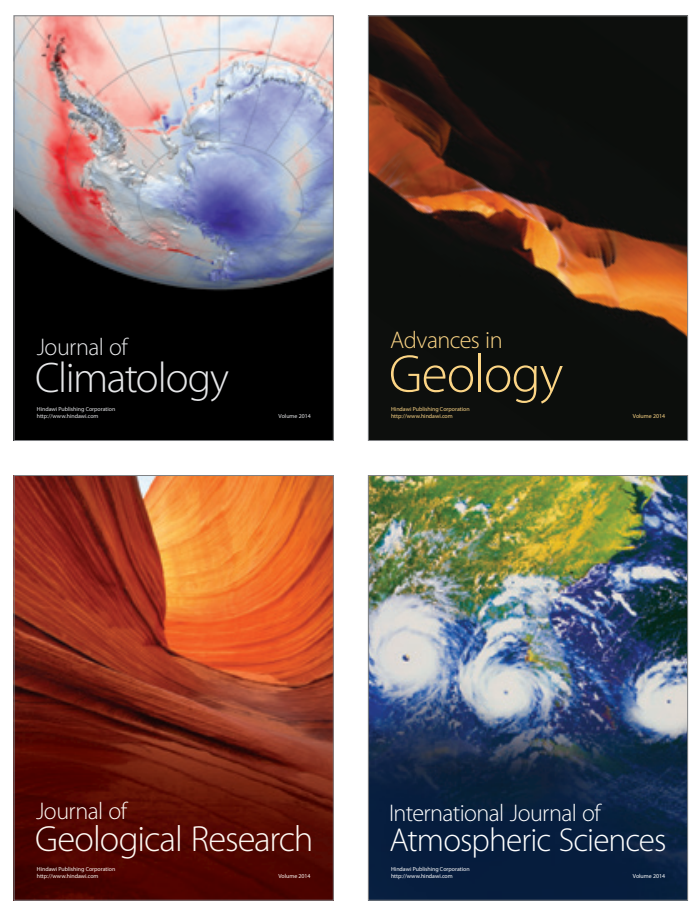
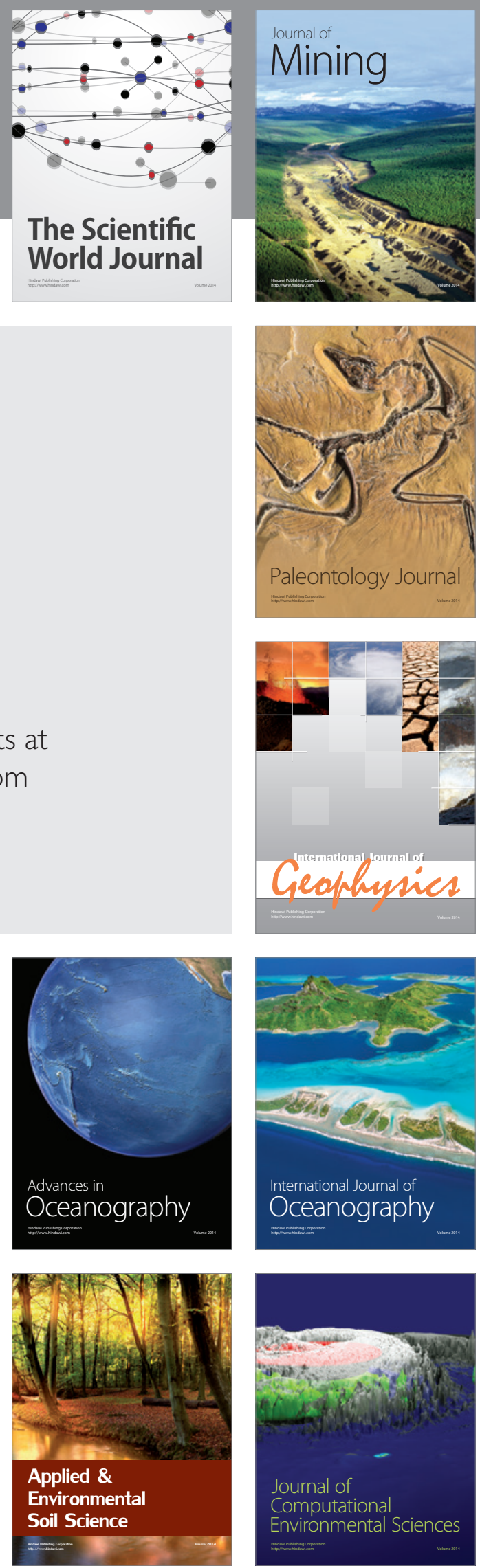